\title{
A Numerical Investigation \\ Into the Length of the Period \\ of the Continued Fraction Expansion of $\sqrt{D}$
}

\author{
By H. C. Williams
}

\begin{abstract}
Let $p(D)$ be the length of the period of the continued fraction expansion of $\sqrt{D}$, where $D$ is a positive square-free integer. In this paper it is suggested that $p(D)=$ $O(\sqrt{D} \log \log D)$ and several tables of numerical results, which support this suggestion, are provided.
\end{abstract}

1. Introduction. Let $\theta$ be any positive irrational and let

$$
\theta=\left[q_{0}, q_{1}, q_{2}, \ldots, q_{n-1}, \theta_{n}\right]
$$

be the continued fraction expansion of $\sqrt{D}$. If $\theta=\sqrt{D}$, where $D$ is a square-free positive integer, then

$$
\sqrt{D}=\left[q_{0}, \overline{q_{1}, q_{2}, \ldots, q_{p}}\right],
$$

where $p=p(D)$ is the length of the shortest period in this expansion of $\sqrt{D}$. In Stanton, Sudler, and Williams [8] it is shown that if $D>7$, then

$$
p(D)<.72 \sqrt{D} \log D .
$$

In Cohn [2] this result is improved to

$$
p(D)<c D^{1 / 2} \log D+O\left(D^{1 / 2}\right)
$$

where $c=7 / 2 \pi^{2} \approx .3546$.

The brief numerical study on the size of $p(D)$ in Beach and Williams [1] seems to indicate that these bounds are not very good for larger values of $D$. The purpose of this note is to suggest why this should be the case and to provide numerical evidence for the belief that $p(D)$ can be bounded by an expression of the form $C D^{1 / 2} \log \log D$, where $C$ is a constant.

2. Theoretical Considerations. Let $\theta=\sqrt{D}$ and let $\varepsilon_{0}$ be the fundamental unit of $Q(\sqrt{D})$. From Williams and Broere [9], we have

$$
\varepsilon_{0}=\prod_{i=1}^{p} \theta_{i},
$$

unless some $Q_{r}=4$. If this latter case occurs, there must exist a pair of odd integers $x, y$ such that $x^{2}-D y^{2}=4$ is solvable. Thus, we must have $D \equiv 5(\bmod 8)$; also

$$
\varepsilon_{0}^{3}=\prod_{i=1}^{p} \theta_{i}
$$

Received October 1, 1979; revised August 5, 1980.

1980 Mathematics Subject Classification. Primary 10-04, 10A32. 
Now, from (2.8) of [8], we have

$$
\prod_{i=1}^{p} \theta_{i}>\alpha^{p}
$$

where $\alpha=(1+\sqrt{5}) / 2$. Hence, if we define $\lambda=1 / 3$ when $D \equiv 5(\bmod 8)$ and $\lambda=1$ otherwise, we have $R>\lambda p \log \alpha$, where $R=\log \varepsilon_{0}$ is the regulator of $Q(\sqrt{D})$.

Levy [3] has shown that, for almost all irrationals $\theta$, we have

$$
\lim _{n \rightarrow \infty} \sqrt[n]{\theta_{1} \theta_{2} \theta_{3} \cdots \theta_{n}}=e^{m}
$$

where $m=\pi^{2} / 12 \log 2 \approx 1.18656911$. Thus, if $p$ is large, we would expect $R \approx$ $\lambda$ pm. For example, when $D=26437680473689$, we have $R \approx 21737796.43$ (Shanks [5]), $p=18331889$ (see [7]), and $R / p \approx 1.185791406$.

If $h$ is the class number of $Q(\sqrt{D})$, it is well known that

$$
2 R h=\sqrt{d} L\left(1, \chi_{d}\right),
$$

where $d$ is the discriminant of $Q(\sqrt{D})$; that is,

$$
d= \begin{cases}D & \text { when } D \equiv 1(\bmod 4) \\ 4 D & \text { otherwise. }\end{cases}
$$

Here

$$
L\left(1, \chi_{d}\right)=\sum_{n=1}^{\infty}\left(\frac{d}{n}\right) \frac{1}{n}=\prod_{q=2}^{\infty}\left(\frac{q}{q-(d / q)}\right),
$$

where $\chi_{d}(n)=(d / n)$ is the Kronecker symbol and the (Euler) product is evaluated over all the primes $q$. Littlewood [4] showed that if the Extended Riemann Hypothesis $(\mathrm{ERH})$ is true for $\chi_{d}(n)=(d / n)$, then

$$
L\left(1, \chi_{d}\right)<\{1+o(1)\} 2 e^{\gamma} \log \log d,
$$

where $\gamma$ is Euler's constant. Further, Shanks [6] noted that under the same hypotheses,

$$
L_{-D}(1)=\sum_{m=1}^{\infty}\left(\frac{4 D}{m}\right) \frac{1}{m}<\{1+o(1)\} e^{\gamma} \log \log 4 D .
$$

From the Euler product in (3), we see that

$$
L_{-D}(1)= \begin{cases}\frac{1}{2} L\left(1, \chi_{d}\right) & \text { when } D \equiv 1(\bmod 8), \\ \frac{3}{2} L\left(1, \chi_{d}\right) & \text { when } D \equiv 5(\bmod 8) \\ L\left(1, \chi_{d}\right) & \text { otherwise; }\end{cases}
$$

hence, Shanks' result is stronger than Littlewood's except when $D \equiv 1(\bmod 8)$.

If we define

$$
f(D)= \begin{cases}\sqrt{D} \log \log D & \text { for } D \equiv 1(\bmod 8) \\ \sqrt{D} \log \log 4 D & \text { otherwise }\end{cases}
$$

we have

$$
R h<\{1+o(1)\} \lambda e^{\gamma} f(D)
$$


From the above remarks we would certainly expect that

$$
G(D)=p(D) / f(D)<K_{1}+o(1)
$$

and even that

$$
G(D)<K_{2}+o(1),
$$

where $K_{1}=e^{\gamma} / \log \alpha \approx 3.701223297$ and

$$
K_{2}=e^{\gamma} m=12 e^{\gamma} \log 2 / \pi^{2} \approx 1.501027123 \text {, provided the ERH is true. }
$$

3. Numerical Results. By using the algorithm mentioned in [9], $p(D)$ was evaluated for all square-free $D$ where $2<D<2 \times 10^{7}$. In Table 1 we present only those values of $D$ such that $p(D)>p(E)$ for all $E<D$. We also show the prime factorization of these $D$ values together with $p(D)$ and $G(D)$ in (5).

\begin{tabular}{|c|c|}
\hline 2 & 2 \\
\hline 3 & 3 \\
\hline 7 & 7 \\
\hline 13 & 13 \\
\hline 19 & 19 \\
\hline 31 & 31 \\
\hline 43 & 43 \\
\hline 46 & 2.23 \\
\hline 94 & 2.47 \\
\hline 139 & 139 \\
\hline 151 & 151 \\
\hline 166 & 2.83 \\
\hline 211 & 211 \\
\hline 331 & 331 \\
\hline 421 & 421 \\
\hline 526 & 2.263 \\
\hline 571 & 571 \\
\hline 604 & 151 \\
\hline 631 & 631 \\
\hline 751 & 751 \\
\hline 886 & 2.443 \\
\hline 919 & 919 \\
\hline 1291 & 1291 \\
\hline 1324 & 331 \\
\hline 1366 & 2.683 \\
\hline 1516 & 379 \\
\hline 1621 & 1621 \\
\hline 1726 & 2.863 \\
\hline 2011 & 2011 \\
\hline 2311 & 2311 \\
\hline 2566 & 2.1283 \\
\hline 2671 & 2671 \\
\hline 3004 & 751 \\
\hline 3019 & 3019 \\
\hline 3334 & 2.1667 \\
\hline 3691 & 3691 \\
\hline 3931 & 3931 \\
\hline 4174 & 2.2087 \\
\hline 4846 & 2.2423 \\
\hline 5119 & 5119 \\
\hline 6211 & 6211 \\
\hline 6451 & 6451 \\
\hline 6679 & 6679 \\
\hline 6694 & 2.3347 \\
\hline 7606 & 2.3803 \\
\hline 8254 & 2.4127 \\
\hline 8779 & 8779 \\
\hline 8941 & 8941 \\
\hline 9739 & 9739 \\
\hline 9949 & 9949 \\
\hline 0399 & 10399 \\
\hline & 10651 \\
\hline & 2.5387 \\
\hline & 12541 \\
\hline 2919 & 12919 \\
\hline
\end{tabular}

TABle 1

D
PERIOD G(D)

\subsection{2}

1.268574

1.256078

1.009257

0.939114

0.913538

0.930715

1.071314

0.927143

0.828021

0.876523

0.912368

0.938215

0.947446

0.899215

0.857079

0.859239

0.872132

0.928307

0.912086

0.863544

0.940111

0.804128

0.818534

0.879911

0.901798

0.903331

0.971966

0.954351

0.902889

0.905738

0.903403

0.879654

0.925995

0.907828

0.887854

0.914096

0.925506

0.953606

0.949949

0.866183

0.912993

0.906496

0.915922

0.952913

0.921061

0.918119

0.931590

0.902425

0.921824

0.945694

0.958119

0.968478

0.896083

0.915071

$\begin{array}{llrl}\text { D } & \text { FACTORS } & \text { PERIOD } & \text { G(D) } \\ 13126 & 2.6563 & & \\ 15031 & 15031 & 262 & 0.958487 \\ 16669 & 79.211 & 268 & 0.911469 \\ 17341 & 17341 & 280 & 0.900769 \\ 17494 & 2.8747 & 281 & 0.884992 \\ 17614 & 2.8807 & 290 & 0.909037 \\ 18379 & 18379 & 300 & 0.936937 \\ 19231 & 19231 & 322 & 0.982945 \\ 21319 & 21319 & 332 & 0.989114 \\ 23599 & 23599 & 348 & 0.981006 \\ 25939 & 25939 & 352 & 0.939685 \\ 27589 & 47.587 & 374 & 0.949117 \\ 28414 & 2.14207 & 380 & 0.933031 \\ 31606 & 2.15803 & 388 & 0.937773 \\ 32839 & 32839 & 394 & 0.899571 \\ 32971 & 32971 & 400 & 0.894780 \\ 34654 & 2.17327 & 438 & 0.977686 \\ 38119 & 38119 & 440 & 0.956370 \\ 39439 & 39439 & 444 & 0.917183 \\ 39901 & 39901 & 448 & 0.908783 \\ 40429 & 40429 & 449 & 0.905168 \\ 40639 & 40639 & 451 & 0.902845 \\ 42046 & 2.21023 & 456 & 0.910334 \\ 42571 & 42571 & 464 & 0.909638 \\ 43261 & 43261 & 474 & 0.923112 \\ 46006 & 2.23003 & 489 & 0.944193 \\ 48799 & 48799 & 506 & 0.945490 \\ 53299 & 53299 & 544 & 0.985062 \\ 60811 & 60811 & 566 & 0.977858 \\ 61051 & 61051 & 574 & 0.924468 \\ 67846 & 2.33923 & 614 & 0.986821 \\ 72934 & 2.36467 & 618 & 0.939040 \\ 73516 & 18379 & 642 & 0.938725 \\ 76651 & 76651 & 644 & 0.937681 \\ 78094 & 2.39047 & 654 & 0.931348 \\ 78439 & 78439 & 692 & 0.975748 \\ 82471 & 82471 & 696 & 0.979093 \\ 85999 & 85999 & 716 & 0.980770 \\ 90931 & 90931 & 720 & 0.964559 \\ 95131 & 95131 & 734 & 0.954639 \\ 100291 & 100291 & 750 & 0.952358 \\ 102859 & 102859 & 754 & 0.930986 \\ 106591 & 106591 & 790 & 0.962446 \\ 111094 & 2.55547 & 808 & 0.965950 \\ 127219 & 127219 & 834 & 0.975404 \\ 131884 & 32971 & 838 & 0.912178 \\ 133519 & 133519 & 864 & 0.922716 \\ 139591 & 139591 & 876 & 0.929449 \\ 145006 & 2.72503 & 904 & 0.936842 \\ 155299 & 155299 & 944 & 0.958789 \\ 162094 & 2.81047 & 1016 & 0.938340 \\ 166846 & 2.83423 & 1028 & 0.972865 \\ 173671 & 173671 & 1040 & 0.969430 \\ 187366 & 2.93683 & 1106 & 0.980963 \\ 189814 & 2.94907 & 1110 & 0.977781 \\ & & & \\ & & & \end{array}$


TABLE 1 (continued)

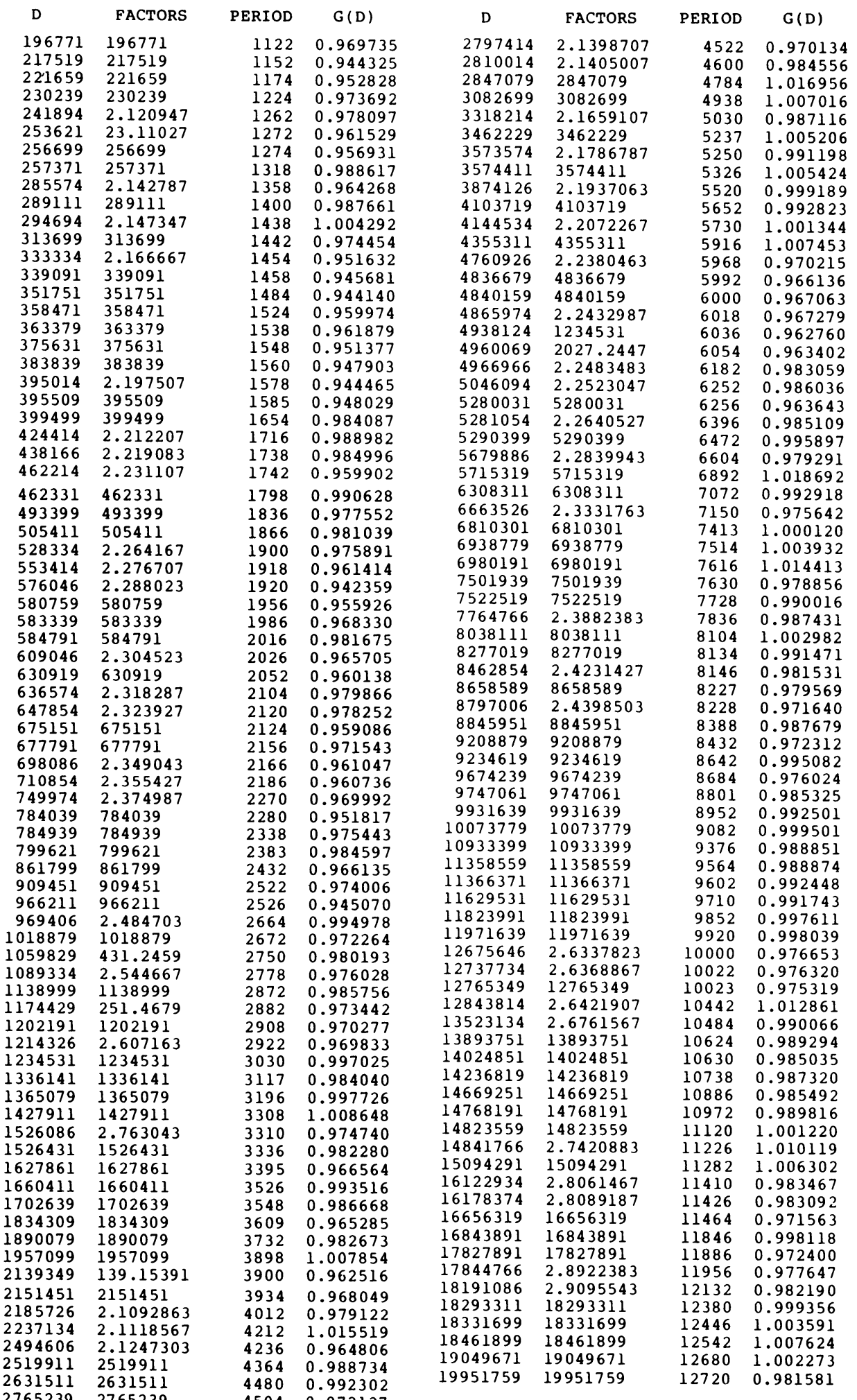


Note that each number $D$ in Table 1 is either $q, 2 q_{1}$, or $q_{1} q_{2}$, where $q$ is a prime and $q_{1}, q_{2}$ are primes of the form $4 k-1$. Since $h$ for $Q(\sqrt{D})$ can be 1 only for such values of $D$, this, in view of (4), is just what we would expect. In order to save computer time, Table 1 was extended up to $5 \times 10^{7}$ by examining only numbers of the above forms. These results are presented in Table 2.

TABLE 2

$\begin{array}{clclcccc}\text { D } & \text { FACTORS } & \text { PERIOD } & \text { G(D) } & \text { D } & \text { FACTORS } & \text { PERIOD } & \text { G (D) } \\ 20041891 & 20041891 & 12942 & 0.996378 & 31277731 & 31277731 & 16638 & 1.016891 \\ 20289091 & 20289091 & 13358 & 1.021884 & 32483851 & 32483851 & 16838 & 1.009129 \\ 21548011 & 21548011 & 13546 & 1.004397 & 34156651 & 34156651 & 17134 & 1.000490 \\ 22365379 & 22365379 & 13642 & 0.992165 & 34225591 & 34225591 & 17272 & 1.007495 \\ 22748014 & 2.11374007 & 13668 & 0.985346 & 37260094 & 2.18630047 & 17648 & 0.985098 \\ 22861276 & 5715319 & 13800 & 0.992302 & 37290031 & 37290031 & 17716 & 0.988482 \\ 23196079 & 23196079 & 13812 & 0.985703 & 37479751 & 37479751 & 17752 & 0.987890 \\ 23345326 & 2.11672663 & 14348 & 1.020554 & 38010751 & 38010751 & 17924 & 0.990219 \\ 23817271 & 23817271 & 14488 & 1.019868 & 38839579 & 38839579 & 18218 & 0.995276 \\ 25609159 & 25609159 & 14632 & 0.991974 & 39803611 & 39803611 & 19002 & 1.025004 \\ 25959781 & 25959781 & 14771 & 0.994360 & 40781911 & 40781911 & 19396 & 1.033181 \\ 26030239 & 26030239 & 14876 & 1.000023 & 43503931 & 43503931 & 19510 & 1.005049 \\ 26796526 & 2.13398263 & 15104 & 1.000187 & 44450701 & 44450701 & 19743 & 1.005774 \\ 27512731 & 27512731 & 15354 & 1.002931 & 44488159 & 44488159 & 19868 & 1.011701 \\ 27892771 & 27892771 & 15514 & 1.006200 & 47710909 & 47710909 & 20121 & 0.988142 \\ 28473454 & 2.14236727 & 15876 & 1.018737 & 48924019 & 48924019 & 20398 & 0.988807 \\ 30343366 & 2.15171683 & 15894 & 0.986810 & 49196359 & 49196359 & 20608 & 0.996119 \\ 30345319 & 30345319 & 16300 & 1.011984 & 49241494 & 2.24620747 & 20826 & 1.006179\end{array}$

In Table 2 no value of $D$ of the form $q_{1} q_{2}$ appears. Also, for each value of $D$ in the table, except 22365379 and 22748014, we have $\left(D / r_{i}\right)=1(i=1,2,3, \ldots, 10)$, where $r_{1}=3, r_{2}=5, r_{3}=7, \ldots, r_{10}=31$, the first ten odd primes. This again is what we would expect. If we examine (3), we see that $L\left(1, \chi_{d}\right)$ will be largest when its leading terms are positive. The larger $L\left(1, \chi_{d}\right)$ is, the larger $R$ and hence $p$ will be. With this in mind, further calculations were performed for values of $D$ $\left(>5 \times 10^{7}\right)$ of the form $q$ and $2 q_{1}$, such that $\left(D / r_{i}\right)=1(i=1,2,3, \ldots, 10)$. In Table 3 we present these calculations for $D=q \equiv-1(\bmod 4)$, in Table 4 for $D=q \equiv 1(\bmod 4)$, and in Table 5 for $D=2 q_{1}$. Each of these tables was computed for all values of such $D$ up to (and in some cases somewhat beyond) $2 \times 10^{9}$. Again, only the values of $D$ for which $p(D)$ is a local maximum are printed.

TABLE 3

$\begin{array}{cccccc}\text { D } & \text { PERIOD } & \text { G (D) } & \text { D } & \text { PERIOD } & \text { G(D) } \\ 51562471 & 20828 & 0.982565 & 82557931 & 27166 & 1.004541 \\ 52022359 & 21128 & 0.992146 & 83256391 & 27360 & 1.007318 \\ 52192891 & 21206 & 0.994124 & 83808631 & 27468 & 1.007842 \\ 52981699 & 21390 & 0.994993 & 85181539 & 27866 & 1.013891 \\ 54467659 & 21654 & 0.992954 & 88720459 & 28170 & 1.003604 \\ 54983839 & 21868 & 0.997883 & 93016291 & 28794 & 1.001060 \\ 55953979 & 21914 & 0.990970 & 96045511 & 28820 & 0.985501 \\ 56283439 & 22084 & 0.995628 & 96910531 & 28866 & 0.982510 \\ 59028979 & 22910 & 1.007717 & 97544899 & 29818 & 1.011496 \\ 59495419 & 22958 & 1.005723 & 101164519 & 29864 & 0.994155 \\ 62290099 & 23526 & 1.006413 & 102808099 & 29986 & 0.989936 \\ 63709759 & 23592 & 0.997536 & 103113739 & 30170 & 0.994483 \\ 64463239 & 23656 & 0.994176 & 104671891 & 30174 & 0.986934 \\ 65014819 & 23686 & 0.991058 & 105947011 & 30566 & 0.993519 \\ 65290471 & 23716 & 0.990144 & 106347151 & 31368 & 1.017602 \\ 67392439 & 24412 & 1.002627 & 108075991 & 31392 & 1.009929 \\ 71338411 & 24822 & 0.989894 & 109032751 & 31724 & 1.015971 \\ 73124731 & 24934 & 0.981721 & 113215411 & 31962 & 1.003873 \\ 73461259 & 25354 & 0.995889 & 116189371 & 32358 & 1.002784 \\ 74382499 & 25398 & 0.991208 & 117480379 & 32446 & 0.999786 \\ 75442579 & 25962 & 1.005829 & 117678031 & 32652 & 1.005260 \\ 76746679 & 26156 & 1.004401 & 119749939 & 32862 & 1.002643 \\ 77288311 & 26332 & 1.007489 & 121135459 & 32878 & 0.997186 \\ 78986779 & 26946 & 1.019456 & 121935199 & 33072 & 0.999665\end{array}$


TABLE 3 (continued)

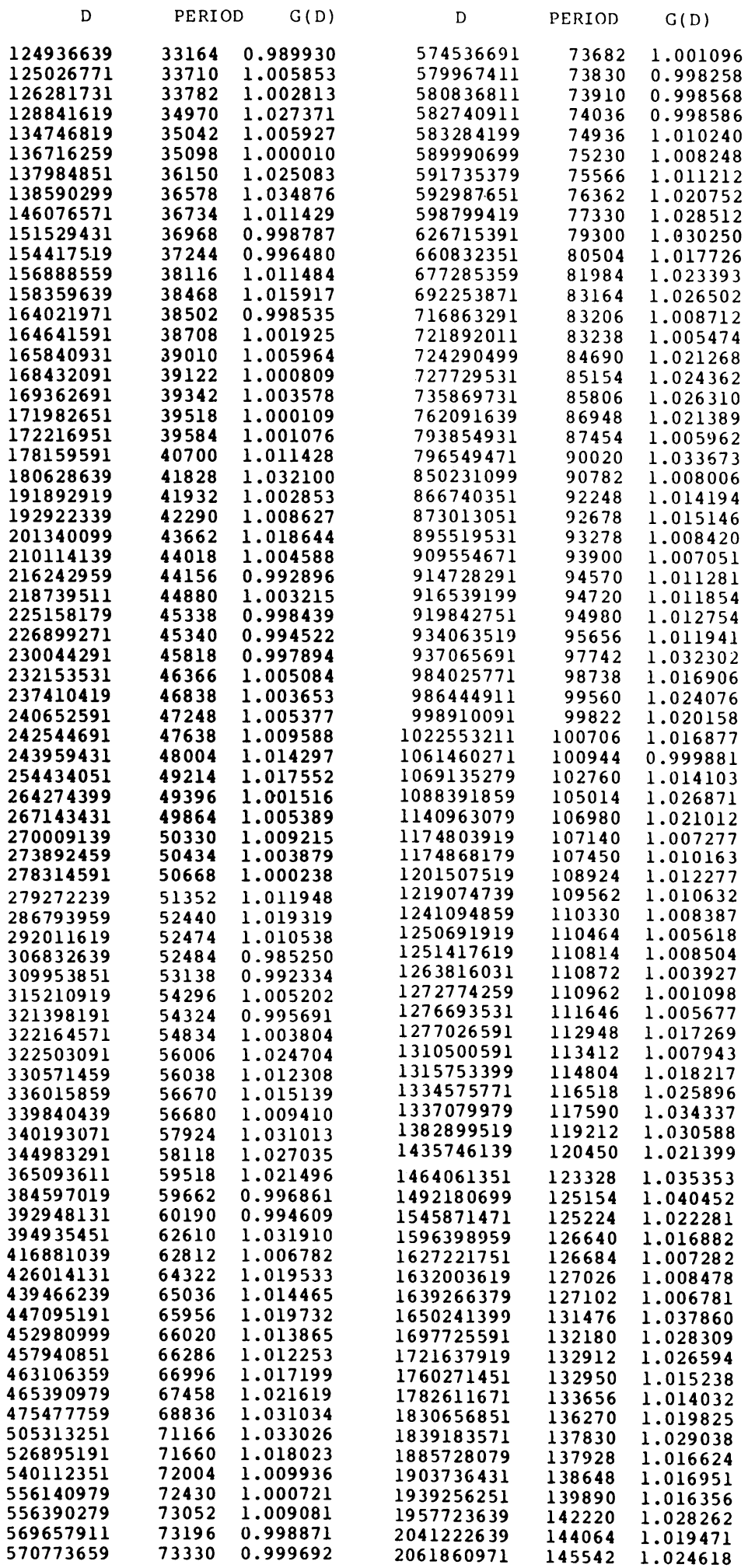


TABLE 4

\begin{tabular}{|c|c|c|c|c|c|}
\hline D & PERIOD & $G(D)$ & D & PERIOD & $G(D)$ \\
\hline 53255029 & 20899 & 0.969567 & 395347261 & 58949 & 0.971050 \\
\hline 53458981 & 20993 & 0.972003 & 395479309 & 60929 & 1.003493 \\
\hline 54632341 & 21189 & 0.970114 & 416813461 & 61583 & 0.987166 \\
\hline 55749541 & 21513 & 0.974681 & 419699149 & 62197 & 0.993469 \\
\hline 60609949 & 21689 & 0.941050 & 433164229 & 63909 & 1.004334 \\
\hline 61419829 & 21699 & 0.935039 & 461090029 & 63961 & 0.973305 \\
\hline 61856701 & 22607 & 0.970600 & 477948949 & 65951 & 0.985186 \\
\hline 62982781 & 22953 & 0.976298 & 478568869 & 66437 & 0.991784 \\
\hline 65271229 & 23241 & 0.970460 & 483892501 & 66911 & 0.993182 \\
\hline 66375709 & 23307 & 0.964803 & 488064901 & 67321 & 0.994857 \\
\hline 66792301 & 23335 & 0.962840 & 509362309 & 67583 & 0.976991 \\
\hline 68463781 & 23581 & 0.960628 & 510949429 & 69069 & 0.996873 \\
\hline 69118261 & 24323 & 0.985990 & 538544029 & 69695 & 0.979016 \\
\hline 72280261 & 24789 & 0.981894 & 544327501 & 71319 & 0.996331 \\
\hline 74563141 & 25181 & 0.981506 & 554156989 & 72057 & 0.997402 \\
\hline 76653229 & 25249 & 0.970184 & 559701949 & 72295 & 0.995577 \\
\hline 77658109 & 25527 & 0.974281 & 559956541 & 72571 & 0.999144 \\
\hline 77835949 & 25683 & 0.979076 & 590067349 & 73087 & 0.979462 \\
\hline 78940261 & 26411 & 0.999519 & 594811309 & 75897 & 1.012933 \\
\hline 84482941 & 26637 & 0.973310 & 623957629 & 75969 & 0.989218 \\
\hline 86262829 & 26973 & 0.975019 & 635189461 & 77911 & 1.005226 \\
\hline 90853669 & 26997 & 0.950071 & 642187309 & 77957 & 1.000160 \\
\hline 91050829 & 27369 & 0.962084 & 662283301 & 78025 & 0.985273 \\
\hline 92408 & 27971 & 0.975747 & 664057549 & 79429 & 1.001621 \\
\hline 92915 & 28871 & 1.004281 & 690716581 & 79605 & 0.983699 \\
\hline 99890389 & 28965 & 0.970565 & 707740069 & 79833 & 0.974225 \\
\hline 100460221 & 29243 & 0.977004 & 711035389 & 80255 & 0.977035 \\
\hline 100685341 & 29685 & 0.990624 & 721277701 & 81365 & 0.983281 \\
\hline 108168 & 30147 & 0.969449 & 730905421 & 82761 & 0.993346 \\
\hline 108414541 & 30343 & 0.974604 & 733665949 & 83019 & 0.994510 \\
\hline 109582 & 30411 & 0.971396 & 757946029 & 83511 & 0.983774 \\
\hline 109866901 & 30631 & 0.977112 & 758248261 & 83551 & 0.984043 \\
\hline 112609429 & 31035 & 0.977465 & 759586909 & 84313 & 0.992116 \\
\hline 118214581 & 31143 & 0.956551 & 773509669 & 84533 & 0.985447 \\
\hline 11915 & 31155 & 0.953002 & 787094389 & 84639 & 0.977878 \\
\hline 119 & 32253 & 0.986311 & 794812789 & 85099 & 0.978266 \\
\hline 121872661 & 32535 & 0.983694 & 800466781 & 85523 & 0.979559 \\
\hline 122515 & 33365 & 1.006050 & 800547829 & 86895 & 0.995221 \\
\hline 1267 & 33465 & 0.991349 & 813539269 & 87149 & 0.989893 \\
\hline 127165789 & 33609 & 0.994089 & 826006861 & 88871 & 1.001580 \\
\hline 137228701 & 34235 & 0.973540 & 852510541 & 89801 & 0.995741 \\
\hline 137870749 & 34435 & 0.976869 & 868915909 & 92353 & 1.014040 \\
\hline 70469 & 34969 & 0.981770 & 929633461 & 93741 & 0.994112 \\
\hline 142308709 & 35009 & 0.977032 & 942744709 & 94199 & 0.991794 \\
\hline 146129701 & 35093 & 0.966065 & 81 & 94 & 0.991157 \\
\hline 146748781 & 35245 & 0.968133 & 981114709 & 97069 & 1.001243 \\
\hline 147041101 & 35767 & 0.981462 & 1021948981 & 98089 & 0.990754 \\
\hline 147280501 & 35869 & 0.983435 & 1027927861 & 98419 & 0.991107 \\
\hline 150407581 & 35941 & 0.974773 & 21 & 98891 & 2353 \\
\hline 152513 & 36581 & 0.985033 & 1036649821 & 100845 & 1.011131 \\
\hline 1589 & 36587 & 0.964253 & 1053014869 & 100977 & 1.004328 \\
\hline 160 & 37821 & 0.991452 & 1060023589 & 101223 & 1.003344 \\
\hline 163384621 & 37945 & 0.986070 & 03941 & 101255 & 0.990437 \\
\hline 166840909 & 38203 & 0.982101 & 1116143989 & 101683 & 0.981502 \\
\hline 170493229 & 38455 & 0.977588 & 1134596941 & 103337 & 0.989087 \\
\hline 173826181 & 39081 & 0.983620 & 1160379949 & 105909 & 1.002053 \\
\hline 177739501 & 39767 & 0.989448 & 1186354621 & 107933 & 1.009638 \\
\hline 183931141 & 41121 & 1.005208 & 1230572821 & 110741 & 1.016585 \\
\hline 197142709 & 42161 & 0.994380 & 1289956669 & 111311 & 0.997344 \\
\hline 204895909 & 42867 & 0.991101 & 1301211781 & 112117 & 1.000086 \\
\hline 212672821 & 43909 & 0.995861 & 1304158909 & 113671 & 1.012769 \\
\hline 217235461 & 44627 & 1.001118 & 1385195821 & 113699 & 0.982090 \\
\hline 226175821 & 45567 & 1.001150 & 1397731141 & 116007 & 0.997393 \\
\hline 236610949 & 45837 & 0.983914 & 1432794661 & 116593 & 0.989739 \\
\hline 236 & 47297 & 1.015077 & 1442507509 & 117483 & 0.993835 \\
\hline 251342701 & 47695 & 0.992384 & 1492904869 & 119175 & 0.990500 \\
\hline 262831501 & 48065 & 0.977287 & 1506539941 & 119421 & 0.987915 \\
\hline & 48395 & 0.977537 & 1517705149 & 119617 & 0.985786 \\
\hline 274963789 & 51973 & 1.032431 & 1541965429 & 121121 & 0.990073 \\
\hline 303728461 & 52161 & 0.984335 & 1553226229 & 121357 & 0.988297 \\
\hline 309156901 & 52843 & 0.988136 & 1566687301 & 125153 & 1.014698 \\
\hline 316660789 & 53383 & 0.985964 & 1660069909 & 127093 & 1.000202 \\
\hline 321437869 & 54795 & 1.004259 & 1668111901 & 129747 & 1.018555 \\
\hline 346477069 & 56947 & 1.004102 & 1760056741 & 130317 & 0.995195 \\
\hline 373293229 & 57637. & 0.977951 & 1769158141 & 133575 & 1.017373 \\
\hline 38 & 583 & 0.980381 & 1855452421 & 137415 & 1.021306 \\
\hline 382399021 & 58919 & 0.987360 & 1895437261 & 140655 & 1.033991 \\
\hline
\end{tabular}


TABLE 5

\begin{tabular}{|c|c|c|c|c|c|}
\hline D & PERIOD & $G(D)$ & D & PERIOD & $G(D)$ \\
\hline $\begin{array}{l}51013174 \\
52184014 \\
52796734 \\
54487294 \\
55839694 \\
59321326 \\
60029566 \\
63437014 \\
63443494 \\
65259286 \\
66140974 \\
67020886\end{array}$ & $\begin{array}{l}21042 \\
21360 \\
21616 \\
21696 \\
22536 \\
22776 \\
23004 \\
23322 \\
23394 \\
23434 \\
23696 \\
24534\end{array}$ & $\begin{array}{l}0.998179 \\
1.001431 \\
1.007327 \\
0.994694 \\
1.020176 \\
0.999266 \\
1.003088 \\
0.988312 \\
0.991310 \\
0.978612 \\
0.982705 \\
1.010525\end{array}$ & $\begin{array}{l}328257526 \\
335152126 \\
335962246 \\
352467886 \\
354601846 \\
371087326 \\
379441126 \\
398301814 \\
402964006 \\
405675526 \\
418270966 \\
420160126\end{array}$ & $\begin{array}{l}55110 \\
56836 \\
57054 \\
57112 \\
58466 \\
59312 \\
60538 \\
60566 \\
61390 \\
61770 \\
62282 \\
63160\end{array}$ & $\begin{array}{l}0.999158 \\
1.019464 \\
1.022101 \\
0.998150 \\
1.018639 \\
1.009451 \\
1.018559 \\
0.993865 \\
1.001361 \\
1.004083 \\
0.996576 \\
1.008280\end{array}$ \\
\hline 69246934 & 24678 & 0.999418 & 420721414 & 63266 & 1.009277 \\
\hline 71363854 & 24732 & 0.986123 & 425326126 & & 1.009627 \\
\hline 72020974 & 24988 & 0.991618 & 439157086 & 65900 & 3316 \\
\hline 72663886 & 25408 & 1.003660 & 468262414 & 67484 & 1.018779 \\
\hline 74409934 & 25452 & 0.993126 & 487067494 & 69342 & 1.025804 \\
\hline 75619294 & 26068 & 1.008715 & 515758006 & 69642 & 1.000304 \\
\hline $\begin{array}{l}79420366 \\
81829654\end{array}$ & $\begin{array}{l}26160 \\
26894\end{array}$ & 0.986921 & 517107046 & 70094 & 1.005442 \\
\hline 85433734 & 27134 & $\begin{array}{l}0.999051 \\
0.985750\end{array}$ & $\begin{array}{l}52247 \\
53540\end{array}$ & & $\begin{array}{l}856 \\
933\end{array}$ \\
\hline 87163246 & 27276 & 0.980692 & 544115014 & $\begin{array}{l}71964 \\
72370\end{array}$ & $\begin{array}{l}1.013933 \\
1.011216\end{array}$ \\
\hline 87322174 & 27732 & 0.996149 & 548033014 & 73510 & 1.023355 \\
\hline 88986934 & 28178 & 1.002334 & 575251966 & 73748 & 1.001350 \\
\hline 91358446 & 28728 & 1.008096 & 575903134 & 73 & 1.001581 \\
\hline 98073166 & 28816 & 0.974781 & 600950494 & 74 & 0.991699 \\
\hline 98205286 & 29014 & 0.980795 & 616 & 75 & 0.987677 \\
\hline 99553294 & 29636 & 0.994786 & 617808 & 75 & 0.989290 \\
\hline 100993006 & 29932 & 0.997293 & 620858254 & 75872 & 0.990492 \\
\hline 101044414 & 30168 & 1.004891 & 626545726 & 75992 & 0.987410 \\
\hline 103345246 & 30720 & 1.011438 & 633787774 & 76448 & 0.9 \\
\hline 111039646 & 30724 & 0.974715 & 637951246 & 77 & 1.001245 \\
\hline 669286 & 31026 & 0.981424 & 643888774 & 79 & 1.02 \\
\hline 112283614 & 31424 & 0.991199 & 671879014 & 81738 & 1.024541 \\
\hline 346 & 32018 & 0.999705 & 698613 & & 1.0 \\
\hline & 32688 & 1.018245 & 726654646 & 84282 & 1.014644 \\
\hline & 32880 & 1.005338 & 728538574 & 85520 & 1.028176 \\
\hline $\begin{array}{l}53694 \\
03926\end{array}$ & 33280 & 0.998287 & 738915046 & 86494 & 1.032342 \\
\hline & 35178 & 1.035717 & 788809774 & 87724 & 1.012384 \\
\hline 19526 & 35282 & 1.007527 & 796562974 & 87 & 1.010194 \\
\hline 92414 & 36248 & 1.025532 & 803297 & 89 & 1.018195 \\
\hline 15258 & 36432 & 0.980799 & 84278 & 18 & 1.031941 \\
\hline $\begin{array}{l}153 \\
158\end{array}$ & 38016 & 1.021187 & 86 & 92 & 1.0 \\
\hline $\begin{array}{l}1681158726 \\
1611587\end{array}$ & $\begin{array}{l}38072 \\
38738\end{array}$ & $\begin{array}{l}1.004251 \\
1.013833\end{array}$ & 893 & & 1. \\
\hline 169739014 & 39054 & 0.995091 & $\begin{array}{l}907 \\
908\end{array}$ & $\begin{array}{l}93406 \\
95538\end{array}$ & 1.003113 \\
\hline 1715 & 39268 & 0.994 & 908 & & 290 \\
\hline 172 & 39886 & 1.006953 & $\begin{array}{l}910511 \\
946711\end{array}$ & $\begin{array}{l}95930 \\
96308\end{array}$ & 1.0 \\
\hline 173 & 40214 & 1.012544 & 970397 & $\begin{array}{l}9 \\
9\end{array}$ & 1.0 \\
\hline 177566614 & 40242 & 1.001768 & 989718766 & $\begin{array}{l}97786 \\
98004\end{array}$ & $\begin{array}{l}1.014356 \\
1.006354\end{array}$ \\
\hline 177 & 40298 & 1.002254 & 99 & 99476 & 1.017977 \\
\hline 82871406 & 41276 & 1.012012 & 998660854 & & 1.017222 \\
\hline & 41344 & 1.012321 & 102214 & 100 & 1.017287 \\
\hline 19 & 41378 & 0.991765 & 1025302534 & 101 & 1.021241 \\
\hline & 41800 & 1.001671 & 1071565174 & 101 & 1.001977 \\
\hline $96-2>$ & 42420 & 1.003448 & 1085931526 & 10? & 1.006139 \\
\hline 202 & 43648 & 1.015531 & 1089373366 & 102966 & 1.006378 \\
\hline 27 & 44006 & 0.991029 & 5475086 & 104 & 1.015009 \\
\hline Oyc & 44548 & 1.001702 & 1138979326 & 105800 & 1.010654 \\
\hline 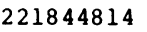 & 44564 & 0.9889 & 2326 & 106130 & 1.011765 \\
\hline $\mathrm{n}^{2}$ & 44788 & 0.9846 & 002694 & 108390 & 1.011438 \\
\hline & 46064 & 1.0065 & & 108490 & 0.997973 \\
\hline 30 & 46356 & 1.006008 & 8366 & 1099 & 1.005276 \\
\hline 239 & 46800 & 0.999366 & 1290271966 & 1106 & 0.991673 \\
\hline ב30? & 47196 & 1.005840 & 1292542486 & 110886 & 0.992513 \\
\hline & 476 & 1.0 & 454366 & & 1.003679 \\
\hline & 476 & 1.0 & & 113388 & 1.006785 \\
\hline & 478 & 0.9973 & 1341438814 & 113456 & 0.996305 \\
\hline & 482 & 0.9 & & 1143 & 1.000443 \\
\hline & & 1.008432 & 136 & 115698 & 1.007658 \\
\hline & & 1.022794 & 1365708094 & 116476 & 1.013434 \\
\hline Je & 5119 & 1.000975 & 1402581574 & 116662 & 1.001239 \\
\hline 1002 & 51288 & 0.995992 & 1406521726 & 117804 & 1.009583 \\
\hline 7273454 & 51484 & 0.982383 & 1424254654 & 118260 & 1.006981 \\
\hline דנכת & 52702 & 1.001669 & 1440761326 & 118464 & 1.002757 \\
\hline 30814 & 54264 & 1.020556 & 1441607974 & 119722 & 1.013100 \\
\hline 0143 & 543 & 0.9989 & 14569624 & 1199 & 1.009211 \\
\hline 19852894 & 54800 & 1.006914 & 1467125566 & 120360 & 1.009350 \\
\hline
\end{tabular}


TABLE 5 (continued)

$\begin{array}{cccccc}\text { D } & \text { PERIOD } & \text { G(D) } & \text { D } & \text { PERIOD } & \text { G(D) } \\ 1475776054 & 121126 & 1.012707 & 1687335094 & 132490 & 1.033978 \\ 1524382414 & 122088 & 1.003881 & 1798654894 & 132856 & 1.003331 \\ 1537069174 & 122206 & 1.000577 & 1799775286 & 133022 & 1.004263 \\ 1545714934 & 124850 & 1.019280 & 1816733494 & 136858 & 1.028254 \\ 1546343254 & 125110 & 1.021190 & 1896380326 & 137390 & 1.009731 \\ 1569268726 & 125946 & 1.020264 & 1915369054 & 140016 & 1.023773 \\ 1595259766 & 127426 & 1.023570 & 2015561326 & 140576 & 1.001278 \\ 1678290574 & 129424 & 1.012846 & 2024387374 & 143612 & 1.020608\end{array}$

Note that in all of these tables (6) is satisfied.

If we compare our function $G(D)$ to Shanks' [6] upper Littlewood index ULI, we see that we would have $G(D)=K_{2} \cdot \mathrm{ULI} / h$ for almost all $D$. Shanks suggests that the ULI can be expected to slowly increase (on the average) up to an average value of about 3/4. This average value may then begin to deteriorate. He also gives values of $D\left(>10^{19}\right)$ such that the ULI exceeds .7 and becomes as large as .713. It seems from this that we could expect to have many values of $D$ outside of the range of these tables for which $G(D)$ is as large as $3 / 4(1.501)=1.126$.

The largest value of $G(D)$ in our tables here occurs for $D_{1}=1492180699$, where $G\left(D_{1}\right)=1.040452$. It is interesting to note that for $D_{2}=26437680473689$ we have $G\left(D_{2}\right)=1.039159$. This latter $D$ value, however, is a quadratic residue of all primes less than 151 and therefore we would expect it to have a large value for its $L$ function. (In fact, $L\left(1, \chi_{d_{2}}\right)=8.455394$; whereas, $L\left(1, \chi_{d_{1}}\right)=3.831228$.) The reason that $D_{1}$ is stronger than $D_{2}$ here is that $D_{2} \equiv 1(\bmod 8)$ is a pseudosquare and $D_{1} \equiv 3(\bmod 8)$ is not. In [6, p. 275 and Figure 1] Shanks points out why the ULI for the pseudosquares tends to lag behind those of numbers like $D_{1}$. (This tendency will diminish as the values of $D$ become larger.) From this reasoning we would not anticipate finding many values of $D \equiv 1(\bmod 8)$ in the above tables and, in fact, none occurs.

Department of Computer Science

University of Manitoba

Winnipeg, Manitoba, Canada R3T 2 N2

1. B. D. Beach \& H. C. Whliams, "Some computer results on periodic continued fractions," Proc. Second Louisiana Conference on Combinatorics, Graph Theory and Computing, Baton Rouge, La., 1971, pp. 133-146.

2. J. H. E. CoHN, "The length of the period of the simple continued fraction of $d^{1 / 2}$," Pacific J. Math., v. 71, 1977, pp. 21-32.

3. PAul Lévy, "Sur le développement en fraction continue d'un nombre choisi au hasard," Compositio Math., v. 3, 1936, pp. 286-303.

4. J. E. Litrtewood, “On the class-number of the corpus $P(\sqrt{-k})$," Proc. London Math. Soc., v. 28, 1928, pp. 358-372.

5. DANiel Shanks, "The infrastructure of a real quadratic field and its applications," Proc. Boulder Symposium, August 1972, Univ. of Colorado, Boulder, 1972, pp. 217-224.

6. Daniel Shanks, Systematic Examination of Littlewood's Bounds on $L(1, \chi)$, Proc. Sympos. Pure Math., Vol. 24, Amer. Math. Soc., Providence, R.I., 1973, pp. 267 - 283.

7. Daniel Shanks, "Review of UMT File: Two related quadratic surds having continued fractions with exceptionally long periods," Math. Comp., v. 28, 1974, pp. 333-334.

8. R. G. Stanton, G. Sudler, JR. \& H. C. Willinms, "An upper bound for the period of the simple continued fraction for $\sqrt{D}$," Pacific J. Math., v. 67, 1976, pp. 525-536.

9. H. C. Williams \& J. Broere, “A computational technique for evaluating $L(1, \chi)$ and the class number of a real quadratic field," Math. Comp., v. 30, 1976, pp. 887-893. 